\title{
Towards ternary optical communication by soft decoding
}

\begin{abstract}
Unlike the conventional $2 \mathrm{M}$ level soft decision, a new soft decoding technique is proposed on optical ternary system. It is shown that $(852,742,6)$ code improves at $10 \mathrm{~dB}$ coding gain whilst increasing the code efficiency.
\end{abstract}

Keyword: Soft decoding; Optical communication; Ternary optical communication 